\title{
Numerical Simulation of Cavitating Flows using Overset Mesh
}

\author{
Alok Khaware', Vinay Kumar Gupta ${ }^{1}$, KVSS Srikanth ${ }^{2}$, Mohammed Azhar ${ }^{2}$ \\ ${ }^{1}$ ANSYS Inc. \\ Hinjewadi, Pune, India \\ Alok.khaware@ansys.com; Vinaykumar.gupta@ansys.com; \\ ${ }^{2}$ ANSYS Inc. \\ Hinjewadi, Pune, India \\ Kvss.srikanth@ansys.com; Mohammed.azhar@ansys.com
}

\begin{abstract}
Modeling cavitation phenomenon can be a challenging prospect for numerical solvers because the available cavitation models require a fine-tuning of model parameters and a good quality mesh in cavitating zones. Cavitation often occurs in complex geometries such as nozzle injection systems, marine propellers or gear pumps and creating a good quality mesh in such geometries need high skill and enough time. Overset mesh simplifies the overall meshing process in complex geometries by allowing separately generated good quality component meshes, which later overlap on each other to form actual flow domain. The aim of this study is to validate the accuracy of the overset method for cavitating flow problems using a multi-phase RANS flow solver and a homogeneous mixture model. Cavitating flow inside a circular throttle injection system is validated against experiment where mass flow rates at the outlet are compared at different inlet pressures. Flow past a fully submerged wedge-shaped hydrofoil is analysed for different cavitation numbers and multiple angles of attack, where cavity shapes for both overset and non-overset meshes are compared with experimental images. Furthermore, supercavitation phenomenon for the flow past a circular disk is investigated where cavity length and cavity radius are validated against the empirical correlations proposed by different authors. This study also highlights the best practices for Schnerr-Sauer and Zwart-GerberBelamri cavitation models available in ANSYS Fluent with overset mesh.
\end{abstract}

Keywords: Cavitation, Overset mesh, Nozzle cavitation, Supercavitating flow

\section{Introduction}

When the local pressure in a liquid decrease to some point below the saturated vapor pressure of the liquid and subsequently recovers above the vapor pressure, it goes through the process of vaporization, bubble generation and bubble implosion, and this complete phenomenon is called as hydrodynamic cavitation. Cavitation typically occurs when a fluid is passed through a constricted channel at a specific flow velocity such as in nozzles or by mechanical rotation of an object in a liquid such as ship propellers or turbines. The geometrical shape of the channel or the rotating object plays an important role and determines the nature of cavitation. Hydrodynamic cavitation cavern can be developed downstream of the local constriction and generate high energy cavitation bubbles. The subsequent growth and collapse of the cavitation bubbles produce very high energy densities which further results in very high local temperatures and local pressures at the surface of the bubbles for a very short time, although the overall liquid medium remains at ambient conditions. This many a times lead to erosion and damage of the system especially seen on turbine blades and ship propellers. A dimensionless quantity called cavitation number $(\sigma)$ expresses the relationship between the difference of a local absolute pressure from vapor pressure and the kinetic energy per volume and used to characterise the potential of the flow to cavitate.

$$
\sigma=\frac{p-p_{\mathcal{v}}}{\frac{1}{2} \rho v^{2}}
$$

Where, $\rho$ is the density of the fluid, $p$ is the local pressure, $p_{v}$ is the vapor pressure of the fluid and $v$ is the characteristic velocity of the flow

Experimental approach for investigating cavitation phenomenon is mainly audio-visual where hydrophones and wideangle high-speed cameras are used. These approaches are neither time efficient nor always possible in actual application 
field under desired operating conditions. The use of computational fluid dynamics (CFD) simulation to capture cavitation effects in the flow field has gained attention since last two decades in the scientific community as it allowed accurate prediction in quick time. Many works have been done to capture cavitation formation in pumps, propellers, nozzles to increase efficiency, reduce noise or prevent damages. There are studies also performed on supercavitation to reduce drag of under water vehicles. It is very important for a numerical cavitation model to predict the cavity formation accurately under different boundary conditions and for different geometrical shapes, hence once validated, these models can be applied for complex geometries.

\section{Overset Mesh}

Overset method also known as chimera grid method achieved a tremendous success in handling complex geometries and avoiding grid remeshing approach when dealing with moving boundary flow problems as it maintains initial grid quality during simulation. This method simplifies the grid generation process because a complex computational domain can be more easily meshed after it is partitioned into sub-domains and combined with overset interfaces than with patched interfaces. Hole-cutting process is carried out to determine the set of elements which will participate in the solution and deactivate other overlapping elements. Mesh elements in a zone which provide information to any other overlapping cell zones are termed as donors. Receptors, or interpolation cells receive information from corresponding overlap cell zone donors and vice-versa. Figure 1 shows overset interface location for three overlapping zones.

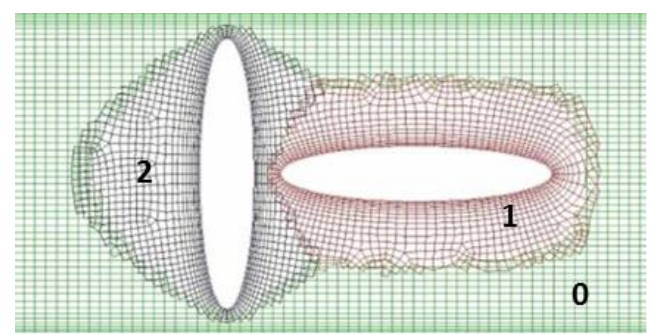

Fig. 1: Overset interface for three overlapping zones.

In this method, the receptor cells receive information from the neighborhood of central donor based on either face connectivity or node connectivity. Solution data gets interpolated whereas gradients are either interpolated or computed. In overset method, interpolation weights are calculated first based on meshing information. Receptor cell values are updated by interpolation method followed by computation of reconstruction and viscous gradients. A linear system is built which establishes the mesh connectivity for each cell and its neighbors from same or different grids. After solving linear system of equations, information on receptor cells are updated from corresponding donors. Information on receptor cell faces are updated from receptor cell and its neighbors on the same grid using standard discretization procedure. Least square based interpolation method in ANSYS Fluent is computationally expensive but provides more accurate results. All the case studied in this paper are done with least square based interpolation.

Figure 2 shows one rectangular receptor cell from Grid-2 overlapping with triangular cells of Grid-1. A nearest cell from the centroid of receptor cell is marked as a central donor. Face connected donors are cells which share faces with central donor as represented by solid lines. Node connected donors are cells which share nodes with central donor. 


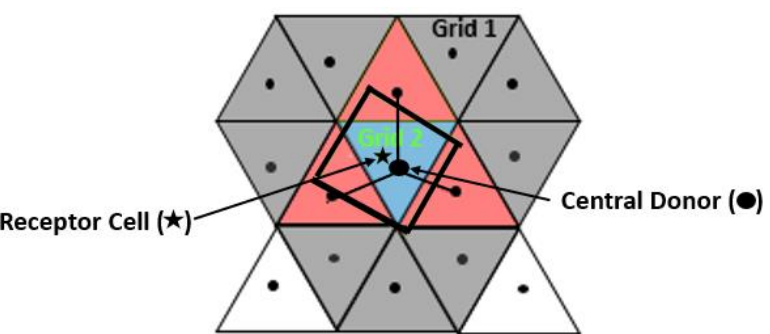

Fig. 2: Overlapping component and background grids and neighbouring donors for a receptor cell.

\section{Cavitation Models}

In multiphase cavitation modelling approach, a basic two-phase cavitation model consists of using the standard viscous flow equations governing the transport of mixture, and a conventional turbulence model. In cavitation, the liquid-vapor mass transfer is governed by the vapor transport equation:

$$
\frac{\partial}{\partial \mathrm{t}}\left(\alpha \rho_{v}\right)+\nabla \cdot\left(\alpha \rho_{v} \vec{V}_{v}\right)=R_{e}-R_{c}
$$

Where, $v$ is vapor phase, $\alpha$ is vapor volume fraction, $\rho_{v}$ is vapor density, $\vec{V}_{v}$ is vapor phase velocity, $R_{e}$ and $R_{c}$ are mass transfer source terms connected to the growth and collapse of the vapor bubbles respectively.

In a flowing liquid with zero velocity slip between the fluid and bubbles, the bubble dynamics equation can be derived from the generalized Rayleigh-Plesset equation as follows

$$
R_{B} \frac{D^{2} R_{B}}{D t^{2}}+\frac{3}{2}\left(\frac{D^{2} R_{B}}{D t}\right)^{2}=\left(\frac{P_{B}-P}{\rho t}\right)-\frac{4 v_{l}}{R_{B}} R_{B}-\frac{2 S}{\rho_{l} R_{B}}
$$

Where, $R_{B}$ is bubble radius, $\sigma$ is liquid surface tension coefficient, $\rho_{l}$ is liquid density, $P_{B}$ is bubble surface pressure and $P$ is local far-field pressure.

In Zwart-Gerber-Belamri cavitation model, it is assumed that all the bubbles in a system have the same size. The total interphase mass transfer rate per unit volume $(R)$ is calculated using the bubble density numbers and the mass change rate of a single bubble [2].

If $P \leq P_{v}$,

When $P \geq P_{v}$,

$$
R_{e}=F_{v a p} \frac{3 \alpha_{n u c}\left(1-\alpha_{v}\right) \rho_{v}}{R_{B}} \sqrt{\frac{2}{3} \frac{\left(P_{v}-P\right)}{\rho_{l}}}
$$

$$
R_{c}=F_{\text {cond }} \frac{3 \alpha_{v} \rho_{v}}{R_{B}} \sqrt{\frac{2}{3} \frac{\left(P-P_{v)}\right.}{\rho_{l}}}
$$

Where, $\alpha_{n u c}$ is the nucleation site volume fraction, $F_{v a p}$ and $F_{c o n d}$ are the evaporation condensation coefficients respectively. Whereas in Schnerr and Sauer cavitation model, vapor volume fraction is connected to the number of bubbles per volume of liquid [2].

If, $P \leq P_{v}$

$$
R_{e}=\frac{\rho_{v} \rho_{l}}{\rho} \alpha(1-\alpha) \frac{3}{R_{B}} \sqrt{\frac{2}{3} \frac{\left(P_{v}-P\right)}{\rho_{l}}}
$$


When, $P \geq P_{v}$

$$
R_{c}=\frac{\rho_{v} \rho_{l}}{\rho} \alpha(1-\alpha) \frac{3}{R_{B}} \sqrt{\frac{2}{3} \frac{\left(P-P_{v}\right)}{\rho_{l}}}
$$

Model constants and their default values for Zwart-Gerber-Belamri model available in ANSYS Fluent are bubble diameter as $1 \mathrm{e}-06 \mathrm{~m}$, nucleation site volume fraction as 0.0005 , evaporation coefficient 500 , condensation coefficient 0.01 , whereas for Schnerr-Sauer model, it is bubble number density as $1 \mathrm{e}+11$ and condensation coefficient as 0.2 .

\section{Numerical Setup}

Homogeneous mixture model with implicit formulation is used for all the case studied in this paper. Pressure based Coupled solver and Pseudo-transient time formulation is used with SST k- $\omega$ turbulence model. Least square cell based gradient method is applied. Pressure is solved as PRESTO, momentum as Second Order, volume fraction and turbulent quantities are as First Order. Zwart-Gerber-Belamri and Schnerr and Sauer cavitation models used where bubble diameter and bubble density are respectively tuned as per solution need. When cavitation vapor formation is under predicted then condensation coefficient is tuned.

\section{Case Studies}

\subsection{Cavitating flow inside a circular throttle injection system 5.1.1. Problem Description}

Cavitation plays an important role to determine the mass flow rate of liquid in nozzle injector outlet. Cavitating flow inside a 3D circular throttle injection system is numerically analysed using overset mesh and non-overset mesh. Liquid phase is the primary phase with a density and viscosity of $825 \mathrm{~kg} / \mathrm{m}^{3}$ and $9.93 \mathrm{e}-04 \mathrm{~kg} / \mathrm{m}-\mathrm{s}$ respectively whereas vapor is the secondary phase with a density, viscosity and vaporization pressure of $0.023 \mathrm{~kg} / \mathrm{m}^{3}, 9.8626 \mathrm{e}-06 \mathrm{~kg} / \mathrm{m}$-s and $3574 \mathrm{~Pa}$ respectively. Inlet pressure is kept constant at 81 bar and outlet pressure is changed according to cavitation number. Experimental mass flow rate at different pressure difference is shown at Table 1. In this testcase, the target variable to evaluate from the CFD simulation is the mass flow rate $(\dot{\mathrm{m}})$ depending on the pressure difference $(\Delta \mathrm{p})$ between inlet and outlet.

Table 1: Experimental mass flow at different pressure difference at inlet and outlet.

\begin{tabular}{|l|l|l|l|l|l|l|l|l|l|l|}
\hline $\mathrm{P}_{\text {out }}[\mathrm{bar}]$ & 71 & 61 & 51 & 41 & 39 & 37 & 35 & 33 & 31 & 21 \\
\hline$\Delta \mathrm{p}[\mathrm{bar}]$ & 10 & 20 & 30 & 40 & 42 & 44 & 46 & 48 & 50 & 60 \\
\hline$\dot{\mathrm{m}}[\mathrm{g} / \mathrm{s}]$ & 4.35 & 6.5 & 8.3 & 9.65 & 9.8 & 10.2 & 10.6 & 10.6 & 10.6 & 10.6 \\
\hline
\end{tabular}

\subsubsection{Geometry and Mesh Preparation}

The geometry has a circular orifice or throttle as shown in the Figure 3. A full 3D model is prepared for overset approach. In this case, only two component meshes are overlapping in orifice zone and no extra background mesh is required Dimensions of nozzle geometry and overlap between components are shown in Figure 3.

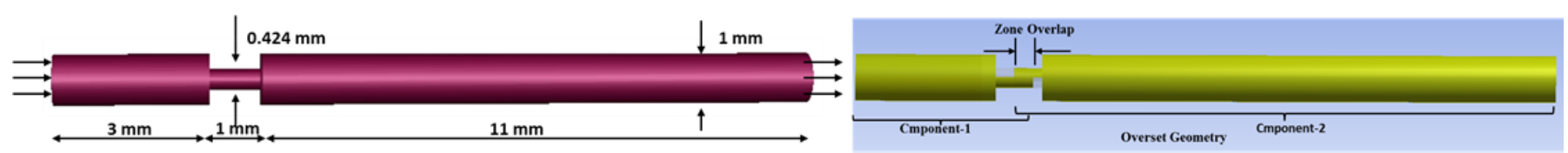

Fig. 3: Nozzle geometry dimensions and zone overlap.

Complete hexahedral mesh elements are generated in 3D model. Three sets of meshes are solved where coarse mesh has a $4.2 \mathrm{M}$ cells, medium mesh has $6 \mathrm{M}$ cells and fine case has $14 \mathrm{M}$ cells. Fine and medium mesh results are very close and medium mesh results are presented in this study. Special care is taken to generate resolved mesh at 
upstream edge of the circular throttle where the onset of cavitation will be observed. Overset interface is intentionally created such a way that vapor formed due to cavitation will cross the interface and overset interpolation accuracy can be verified.
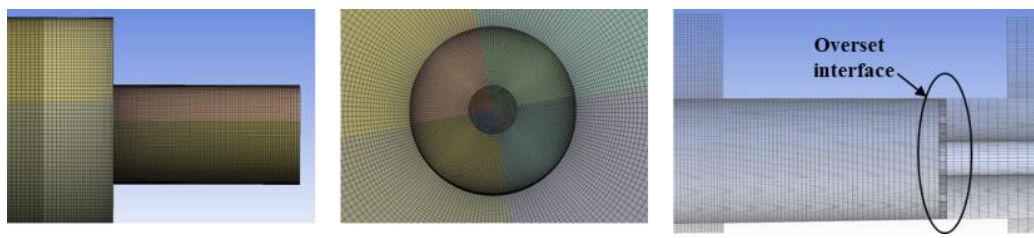

Fig. 4: Complete hexahedral mesh and overset mesh interface.

\subsubsection{Solver Numerics}

Zwart-Gerber-Belamri cavitation model is used for mass transfer formulation with a bubble diameter as $2 \mathrm{e}-06 \mathrm{~m}$ and others as default.

\subsubsection{Results and Discussions}

Cavitation starts when $\Delta p \geq 36$ bar. Contour of the vapor volume fraction in the circular throttle region is shown in the Figure 5 for $\Delta p=60 \mathrm{bar}$. Mass flow rate at outlet is observed for different pressure difference and validated against experimental results [3]. Overset and non-overset mesh results are almost identical.
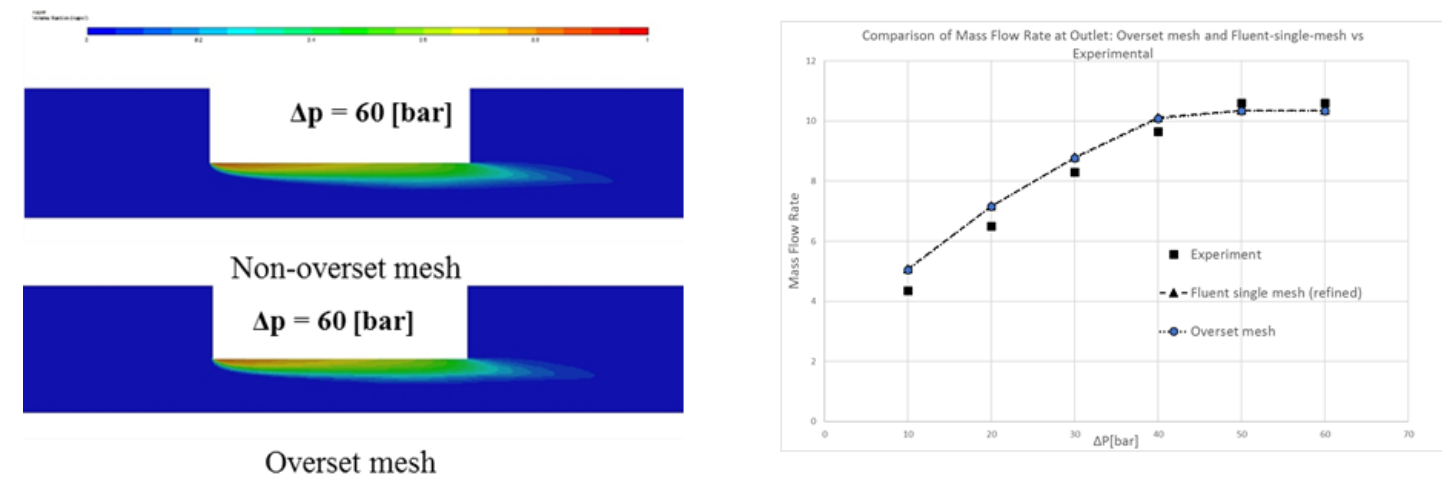

Fig. 5: Volume fraction contours comparison for overset mesh vs non-overset mesh and mass flow rate validation at nozzle outlet.

\subsection{Cavitating flow over a Fully Submerged Wedge \\ 5.2.1. Problem Description}

In this case study, cavitation phenomenon is numerically validated using overset and non-overset mesh at different cavitation numbers and different angle of attacks (AoA) for the flow over a fully submerged wedge object. This case was investigated experimentally by R. W Kermeen (1960) [4] and subsequently validated numerically by S.K.R. Patil et al [5] using tetrahedral non-overset mesh. Flow with a velocity of $9.144 \mathrm{~m} / \mathrm{s}$ is applied at inlet whereas gauge pressure of $0 \mathrm{~Pa}$ is considered at outlet. Water liquid with a density of $998.2 \mathrm{~kg} / \mathrm{m}^{3}$ and viscosity of $0.001003 \mathrm{~kg} / \mathrm{m}-\mathrm{s}$ is used as primary phase whereas secondary phase is water vapor with a density of $0.5542 \mathrm{~kg} / \mathrm{m}^{3}$ and viscosity of $1.34 \mathrm{e}-05 \mathrm{~kg} / \mathrm{m}-\mathrm{s}$. Water vaporization pressure is used as $3540 \mathrm{~Pa}$.

\subsubsection{Geometry and Mesh Preparation}

Wedge length (L) and width (B) are of 3 inches, angle of attack $(\alpha)$ is considered as $8^{0}$ and $15^{\circ}$ for two different solutions respectively. Geometrical dimensions and domain information is presented in Figure 6. A good quality hexahedral mesh is generated with a refinement behind the wedge as shown in Figure 7. 

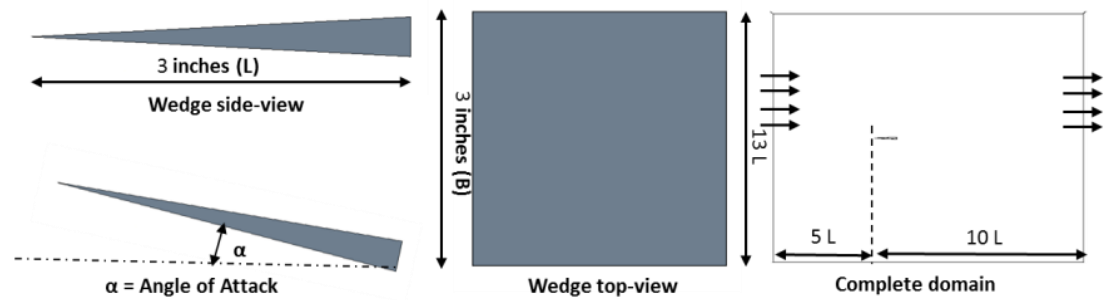

Fig. 6: Geometry and flow domain for wedge hydrofoil case.

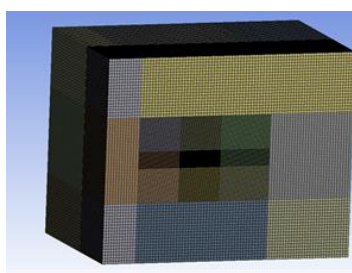

Background mesh

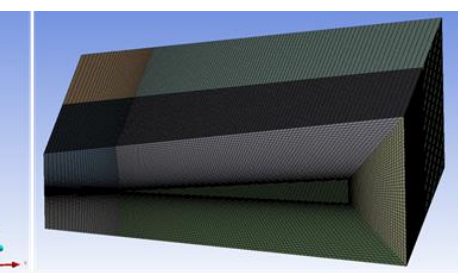

Component mesh

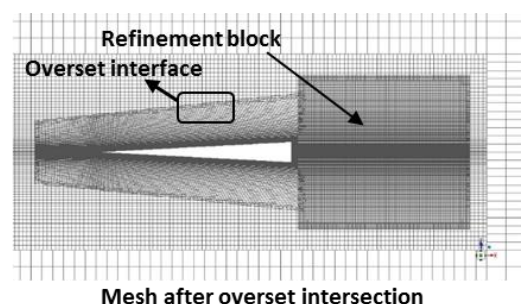

Mesh after overset intersection

Fig. 7: Background and component mesh along with overset mesh interface.

\subsubsection{Solver Numerics}

Pseudo transient time formulation with a time step size of $0.001 \mathrm{~s}$ is applied. Zwart-Gerber-Belamri cavitation model is used for mass transfer formulation with a condensation coefficient as 0.001 and other parameters as default.

\subsubsection{Results and Discussion}

Iso-surface of vapor volume fraction obtained from overset mesh case is compared with experimental image and the results obtained from non-overset mesh at angles of attacks of $8^{0}$ and $15^{0}$, as shown in Figure 8 . As cavitation number is decreases, cavitating area and vapor cavity length increase. It is seen that ANSYS Fluent results agree well with experiment in predicting the cavitating areas on the wedge hydrofoil at different angles of attack and cavitation numbers studied.

Drag and lift coefficients are compared for different cavitation numbers at AOA $8^{0}$ and shown in Figure 9. Lift and drag values have some difference which could be due to turbulence closure as turbulence occurring behind the hydrofoil plays an important role to determine the shape of the cavity. Previous studies have shown that, LES solution may give more close results with experiment [5] which is out of scope from this study.

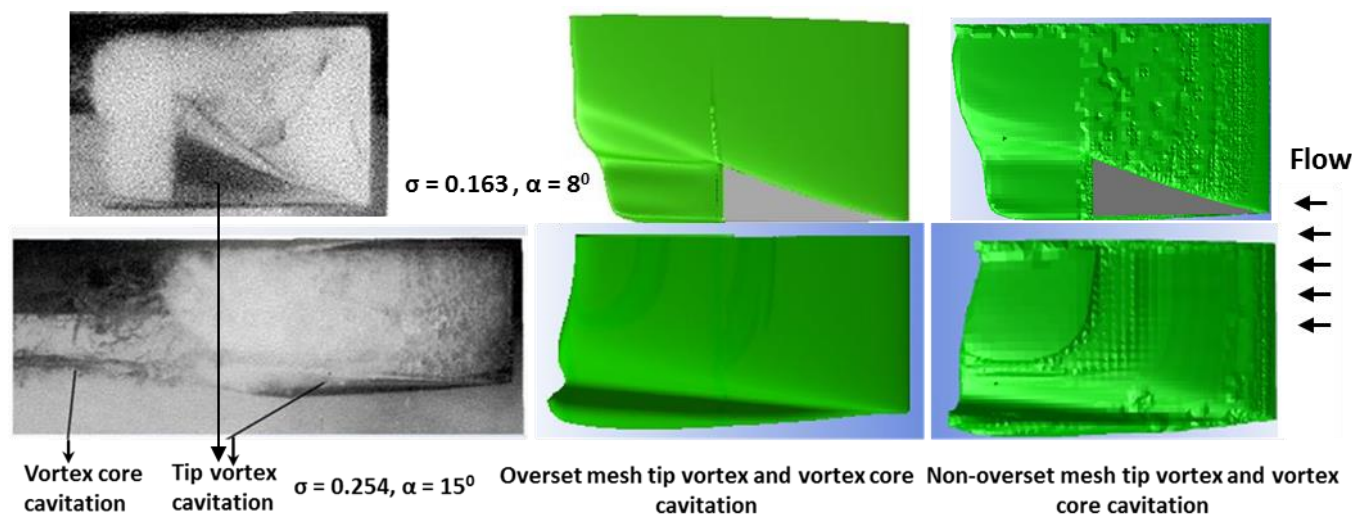

Fig. 8: Comparison of Iso-surface of vapor at different cavitation numbers and angles of attack. 

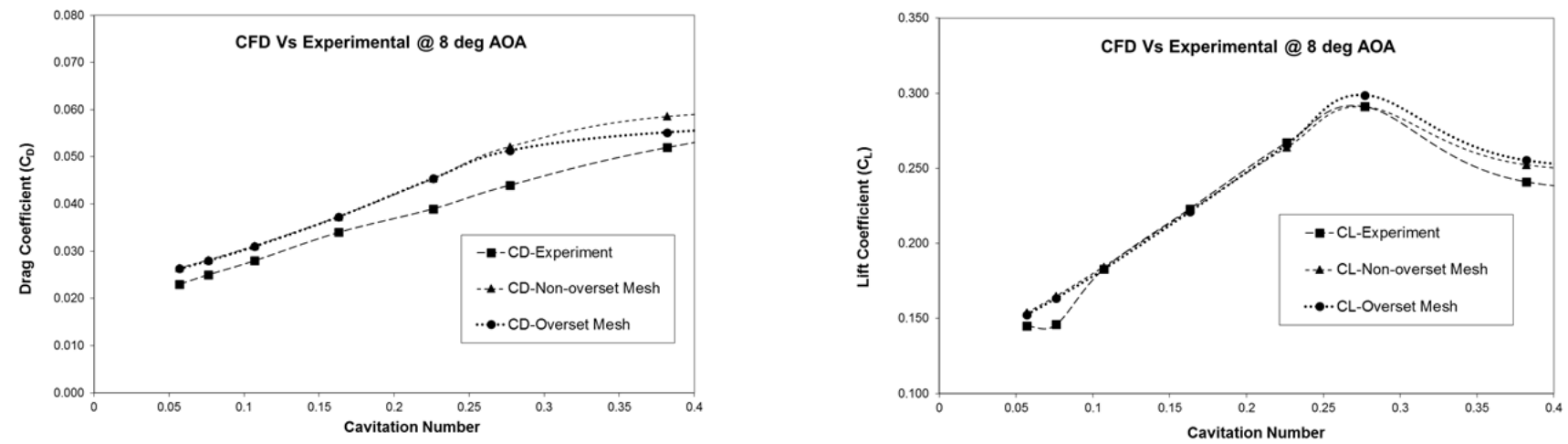

Fig. 9: Validation of drag and lift for overset mesh and non-overset mesh at different cavitation numbers at $8^{0}$ of AOA.

\subsection{Supercavitating flow over Reichardt Plate}

In the case of axisymmetric supercavitation, the cavity shape is like an ellipsoid and expressed as a function of cavitation number, $\sigma$, by the asymptotic formula of Garabedian [6]. Garabedian's formulas for the dimensions of a cavity created by a disk are,

$$
L=d \frac{\sqrt{C_{D}}}{\sigma} \sqrt{\ln \frac{l}{\sigma}}, D=d \sqrt{\frac{C_{D}}{\sigma}}, \quad C_{D}=C_{D_{0}}(1+\sigma) \text { and } C_{D_{0}}=0.827
$$

Where, $\mathrm{d}$ is the diameter of the disk, $C_{D}$ is the drag coefficient of the disk, and $C_{D_{0}}$ is the drag coefficient of the disk at a cavitation number of zero [6,7].

Reichardt (1946) derived semi-empirical formulas [8] to find cavity length which later tasted by Ripken (1955) [7]. Reichardt's formula is valid for $\sigma<0.1$ and can be given as follows

$$
D=d \sqrt{\left(\frac{C_{D}}{\sigma-0.132 \sigma^{8 / 7}}\right)} \text { and } L=D \frac{\sigma+0.008}{\sigma(0.066+1.7 \sigma)}
$$

Waid (1957) performed experimental investigations on shape of a ventilated cavity around a disk and came up with a relation [9] as follows

$$
L=\frac{1.08}{\sigma_{c}^{1.118}} d \text { and } D=\frac{0.534}{\sigma_{c} 0.568}+1
$$

Where, $\sigma_{c}$ is the cavitation number based on cavity pressure.

\subsubsection{Problem Description}

Numerical simulation of the hydrodynamic cavitation for super-cavitating flows over underwater high-speed bodies is targeted in this case study. Supercavitating flow over a circular disk of diameter $0.0635 \mathrm{~m}$ ( $2.5 \mathrm{inch})$ is considered for this validation. A 2-dimensional Axisymmetric approach is considered, and problem is solved using an overset mesh and a nonoverset mesh. Schematic drawing of the domain is shown in Figure 10. Water liquid with a density of $998.2 \mathrm{~kg} / \mathrm{m}^{3}$ is applied as primary phase and vapor with a density of $0.5542 \mathrm{~kg} / \mathrm{m}^{3}$ and vaporization pressure of 3540 as secondary phase. Inlet velocity is equal to $111.12 \mathrm{~m} / \mathrm{s}$ where outlet is set as pressure outlet with 2 atmospheric gauge pressure. 


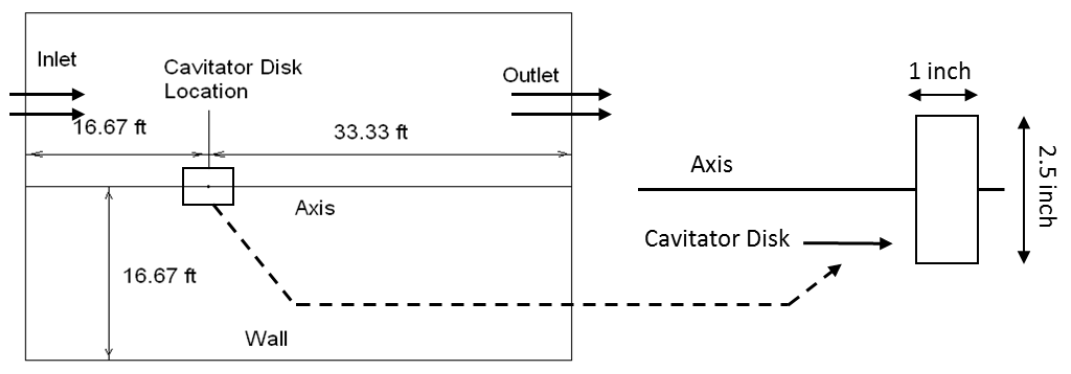

Fig. 10: Schematic drawing of problem description.

\subsubsection{Geometry and Mesh Preparation}

For creating overset mesh, a rectangular background with complete hex mesh is prepared according to the domain. Circular disk is included in component domain. Component geometry is extended such a way that the vapor generated in cavitation process will pass through the overset interface, this will allow to investigate the accuracy of overset interpolation at interface. Non-overset mesh is created with the same mesh resolution as overset so that, the results obtained from both the approaches can be verified. Total mesh elements for non-overset mesh is $1.25 \mathrm{M}$ whereas overset solve cell count is $1.26 \mathrm{M}$.

\subsubsection{Solver Numerics}

Hybrid initialization is used while initializing the flow. This case is first solved without cavitation to establish flow domain and later Schnerr -Sauer cavitation activated and ran with Pseudo time-scale factor of 0.01 for another 10000 iterations. Condensation coefficient is taken 0.005 and turbulence coefficient as 0.39 for Schnerr-Sauer model parameters. Calculated cavitation number $(\sigma)$ is 0.032 .

\subsubsection{Results and Discussion}

Figure 11 presents the variation of cavity radius with respect to cavity length and comparison with the results obtained from Garabedian's formula and Reichardt's formula. The results obtained from overset mesh and non-overset mesh is very identical. Volume fraction of vapor for overset mesh and non-overset mesh is also shown. Resolved mesh behind the disk and disk surface was necessary for this case to achieve expected cavity length.
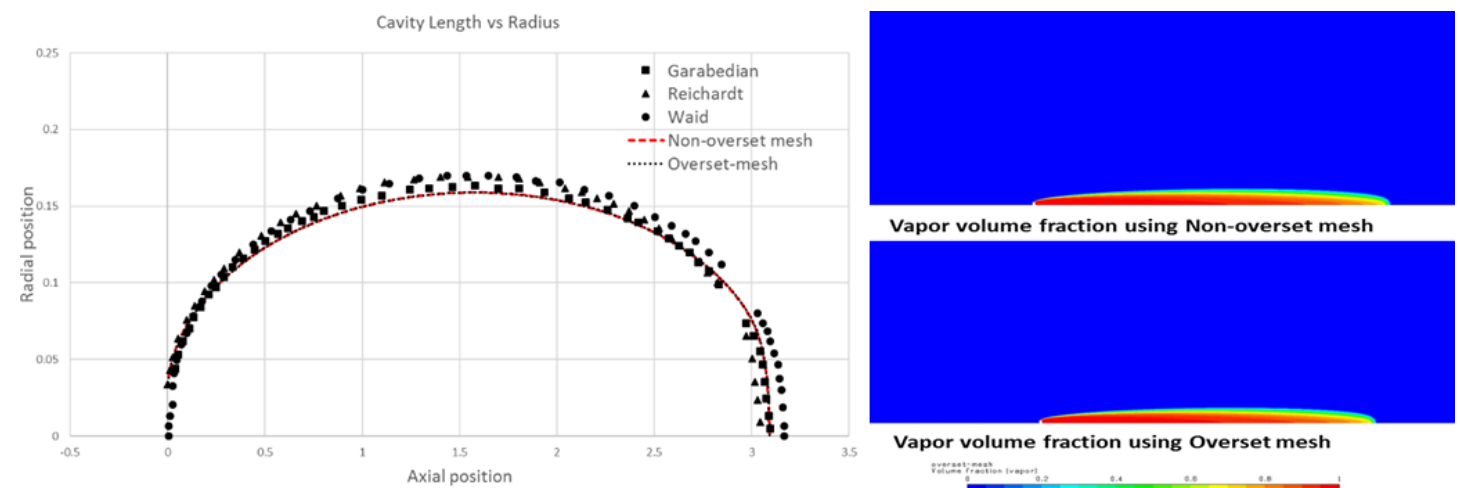

Fig. 11: Cavity length vs cavity radius for overset mesh, non-overset mesh and different analytical correlations. Contours of vapor volume fraction for overset mesh vs non-overset mesh.

\section{Conclusion}

Numerical studies performed in this paper demonstrate that cavitation phenomenon can be numerically modelled accurately using overlapping overset meshes. Three different commonly witnessed cavitation examples have been 
validated in this exercise. The effect of cavitation development in injection nozzles have shown promising results with overset meshing. Sheet cavity formed in the flow over a submerged wedge hydrofoil is well matched with experiment for for both overset and non-overset mesh cases. Supercavitation phenomenon studied in the flow over a circular disk showed showed good match with analytical correlations proposed by different authors.

It is shown that the Schnerr-Sauer and Zwart-Gerber-Belamri cavitation models available in ANSYS Fluent are compatible with overset mesh. This study establishes the fact that the overset mesh approach can be used for cavitating flow cases and results similar to non-overset mesh approach can be expected.

\section{Acknowledgements}

We are thankful to ANSYS Inc. for supporting us to continue this study. Special thanks to Thomas Scheidegger and Srinivas Ramakrishnan for their contribution to development of overset method in ANSYS Fluent and Dr Patrick Sharkey for his review and suggestions.

\section{References}

[1] ANSYS Fluent User Guide, Release 19.2, ANSYS Inc, 2018.

[2] ANSYS Fluent Theory Guide, Release 19.2, ANSYS Inc, 2018.

[3] W. Bauer, U. Iben and M. Voss, "Simulation of cavitating flow in injection systems", VDI Berichte 1846, pp. 10291042, VDI Verlag, 2004.

[4] R. W. Kermeen, "Experimental Investigations of Three-Dimensional Effects on Cavitating Hydrofoils," Department of the Navy Bureau of Ships, Contract Nonr- 220(12), Report No. 47-14, September, 1960.

[5] S. K. R Patil, S. Morton, J. Scherer, A. Egelja-Maruszewski, and M. Davoudabad, "Cavitation Modeling and Validation of a Fully Submerged Wedge," NAFEMS WORLD CONGRESS, 2011, Boston, USA.

[6] P. R. Garabedian, "Calculation of axially symmetric cavities and jets," Pac J Math, vol. 6, pp. 611-684, 1956.

[7] Travis Jon Schauer, "An Experimental Study of a Ventilated Supercavitating Vehicle," A Thesis Submitted to the Faculty of The Graduate School of The University of Minnesota, March 2003.

[8] H. Reichardt, "The Laws of Cavitation Bubbles at Axially Symmetrical Bodies in a Flow," Ministry of Aircraft Production (Great Britain), Reports and Translations, No. 766, 1946.

[9] R. L. Waid, "Cavity Shapes for Circular Disks at Angles of Attack. California Institute of Technology," Report No. E73.4, 1957. 